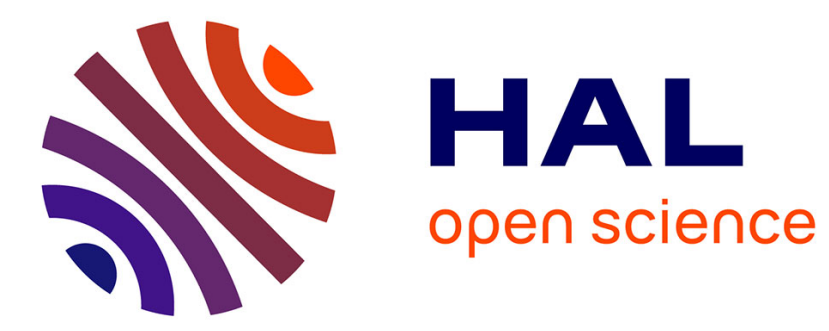

\title{
Fast-Response Superconducting Titanium Bolometric Detectors
}

Jiaqiang Zhong, Wen Zhang, Wei Miao, Dong Liu, Zheng Wang, Wen-Ying Duan, Feng Wu, Kun Zhang, Qijun Yao, Sheng-Cai Shi, et al.

\section{- To cite this version:}

Jiaqiang Zhong, Wen Zhang, Wei Miao, Dong Liu, Zheng Wang, et al.. Fast-Response Superconducting Titanium Bolometric Detectors. IEEE Transactions on Applied Superconductivity, 2019, 29 (5), pp.15. 10.1109/TASC.2019.2900579 . hal-03063842

\section{HAL Id: hal-03063842 https://hal.science/hal-03063842}

Submitted on 14 Dec 2020

HAL is a multi-disciplinary open access archive for the deposit and dissemination of scientific research documents, whether they are published or not. The documents may come from teaching and research institutions in France or abroad, or from public or private research centers.
L'archive ouverte pluridisciplinaire HAL, est destinée au dépôt et à la diffusion de documents scientifiques de niveau recherche, publiés ou non, émanant des établissements d'enseignement et de recherche français ou étrangers, des laboratoires publics ou privés. 


\section{Fast-response superconducting titanium bolometric detectors}

\begin{tabular}{|c|c|}
\hline Journal: & Applied Superconductivity Conference 2018 \\
\hline Manuscript ID & ASC2018-2EOr3B-06.R1 \\
\hline Conference Manuscript Type: & Contributed Manuscript \\
\hline $\begin{array}{r}\text { Date Submitted by the } \\
\text { Author: }\end{array}$ & 23-Jan-2019 \\
\hline Complete List of Authors: & $\begin{array}{l}\text { Zhong, Jia-Qiang; Millimeter and submillimeter lab, Purple Mountain } \\
\text { Observatory } \\
\text { Zhang, Wen; Purple Mountain Observatory, Chinese Academy of } \\
\text { Sciences, Millimeter and submillimeter lab } \\
\text { MIAO, Wei; Purple Mountain Observatory, MM \& SubMM Lab } \\
\text { Liu, Dong; Purple Mountain Observatory, } \\
\text { Wang, Zheng; Purple Mountain Observatory, } \\
\text { Duan, WenYing; Purple Mountain Observatory, SMLAB } \\
\text { Wu, Feng; Purple Mountain Observatory, Chinese Academy of Sciences, } \\
\text { Millimeter and submillimeter lab } \\
\text { Zhang, Kun; Purple Mountain Observatory, SMLAB } \\
\text { Yao, QiJun; Purple Mountain Observatory, SMLAB } \\
\text { Shi, Shengcai; Purple Mountain Observatory, } \\
\text { Wang, Ming-Jye; Academia Sinica, Institute of Astronomy and } \\
\text { Astrophysics (ASIAA) } \\
\text { Pajot, Francois; CNRS-Université Paris Sud-11, IAS }\end{array}$ \\
\hline Keywords: & $\begin{array}{l}\text { Energy-resolving superconducting detectors < Transition-edge sensors } \\
\text { (TES) devices < Superconducting Detectors, Superconducting device } \\
\text { noise < General Electronics < Superconducting Electronics, } \\
\text { Superconducitng photon-counting devices < Transition-edge sensors } \\
\text { (TES) devices < Superconducting Detectors }\end{array}$ \\
\hline
\end{tabular}

\section{SCHOLARONE ${ }^{\text {m }}$ Manuscripts}


1

\title{
Fast-response Superconducting Titanium Bolometric Detectors
}

\author{
JiaQiang Zhong, Wen Zhang, Wei Miao, Dong Liu, Zheng Wang, Wen-Ying Duan, Feng Wu, Kun Zhang, \\ Qijun Yao, Sheng-cai Shi, Ming-Jye Wang, and François Pajot
}

\begin{abstract}
High time resolution optical/Infrared (IR) astronomy will require fast response detectors. In order to meet such requirement, cryogenic optical/IR detectors, such as superconducting tunnel junctions (STJ) and transition-edge sensors (TES), are under development. Of these cryogenic detectors, superconducting titanium (Ti) bolometric detectors, with a transition temperature of around $300 \mathrm{mK}$, based on the electronphonon decoupling mechanism, is promising in fast response. In this paper, we report on the characterization of electro-thermal properties of a small-size $(1 \mu \mathrm{m} \times 1 \mu \mathrm{m} \times 37 \mathrm{~nm})$ superconducting Ti detector. The low frequency noise of $\mathrm{Ti}$ film was fitted by empirical Hooge model. A discrepancy between the Hooge constant of the Ti film and that of normal metals shows disorder in the Ti film. Thermal conductance was estimated extracted via measured current-voltage curves at different bath temperatures. The time constant was calculated as tens of nanoseconds, which was quite fast faster than expected. The fast response potentially enables the high speed optical/IR astronomical observations. Detailed experimental and analytical results are presented.
\end{abstract}

Index Terms-Superconducting detectors, bolometers, electrothermal feedback, noise equivalent power.

\section{INTRODUCTION}

B OLOMETRIC detectors based on weak thermal coupling between an absorber and a heat sink have been widely used in radiation detection. [1]-[3] Among them, superconducting bolometric detectors (a.k.a. TES, transitionedge sensors) have been employed in a large wavelengthrange, from millimeter wave, optical and infrared (IR), to X-rays. [4]-[9] TES detectors with very high sensitivity and relatively large arrays are playing an increasingly important role in millimeter and sub-millimeter astronomy. [10], [11]

This work was supported in part by National Key R\&D program of China under Grant 2017YFA0304003, NSFC under Grants U1731119, 11673073 U1831202, 11473075, Chinese Academy of Sciences (CAS) Key Research Program of Frontier Sciences under Grant QYZDJ-SSW-SLH043, CAS Key Technology Research Team Program under Grant GJJSTD20180003, and by the CAS joint Key Lab for Radio Astronomy.(Corresponding author: Shengcai Shi,e-mail: scshi@pmo.ac.cn.)

JiaQiang Zhong, Wen Zhang, Wei Miao, Dong Liu, Zheng Wang, Wen-Ying Duan, Feng Wu, Kun Zhang, QiJun Yao and Sheng-cai Shi are with the Purple Mountain Observatory and Key Laboratory of Radio Astronomy, Chinese Academy of Sciences, Nanjing 210034, China.(email: jqzhong@pmo.ac.cn; wzhang@pmo.ac.cn; wmiao@pmo.ac.cn; dliu@pmo.ac.cn; zhwang@pmo.ac.cn; w w $\quad$ wduan@pmo.ac.cn; wufeng@pmo.ac.cn; $\quad$ zhangkun@pmo.ac.cn; $\quad$ yqj@mwlab.pmo.ac.cn; scshi@pmo.ac.cn.)

Ming-Jye Wang is with Institute of Astronomy and Astrophysics, Academia Sinica, Taipei, Taiwan.(e-mail: mingjye@asiaa.sinica.edu.tw)

François Pajot is with Institut de Recherche en Astrophysique et Planétologie, Toulouse, France.(e-mail: francois.pajot@irap.omp.eu)

Color versions of one or more of the figures in this paper are available online at http://ieeexplore.ieee.org.

Digital Object Identifier will be inserted here upon acceptance.
For example, the instrument SCUBA-2 on the James Clerk Maxwell Telescope (JCMT) has 10,000 TES and employs time domain multiplexing readout. [11] In ground-based astronomical applications, the requirement of detector sensitivity, i.e., noise equivalent power (NEP), is background-limited at the level of $10^{-17} \mathrm{~W} / \mathrm{Hz}^{0.5}-10^{-16} \mathrm{~W} / \mathrm{Hz}^{0.5}$ at millimeter wavelength and for future space telescopes at the level of $\sim 10^{-20} \mathrm{~W} / \mathrm{Hz}^{0.5}$. [12]-[15] The key to achieve such high sensitivity for TES is small heat capacity. The small heat capacity is obtained by minimizing detector pixel size. [12], [15] At the same time, by using high critical temperature materials such as $\mathrm{Ti}$, fast response photon-number-resolving detection for terahertz/far infrared (THz/FIR) might be possible. [15][17] By coupling to single-mode optical/IR fiber, it is also suitable for optical/IR detection. [18], [19]

Headed for high sensitivity and fast response, we developed small-area Ti TES. [18] [20] In the current paper, we report on the characterization of a Ti TES detector with a micro-bridge of $1 \mu \mathrm{m} \times 1 \mu \mathrm{m} \times 37 \mathrm{~nm}$. The characteristics such as resistive transition, thermal conductance, electro-thermal feedback loop gain, and noise were studied. The time constant was evaluated using the heat capacity and the thermal conductance. The most important result is that the estimated extracted response time constant is smaller shorter than the expected electronphonon relaxation time constant. This result indicates that the overall thermal conductance was higher than the electronphonon one due to diffusion cooling via contacts. $[19],[20]$ [21], [22] The diffusion cooling via contacts takes effect in the small-size detector whereas it is negligible in a largearea detector. A Ti TES detector with relatively large size of $25 \mu \mathrm{m} \times 25 \mu \mathrm{m} \times 51 \mathrm{~nm}$ was then fabricated and tested coupled to a laser source. The optical response using largearea detector was recorded and analyzed to get relaxation time.\{21],[22] [18], [23] The time constant of the small-size detector was smaller shorter than that of the larger one. With high sensitivity, fast response, and low heat capacity, the smallsize Ti TES detector is promising for power detection as well as for PNR detection in astronomical applications.

\section{EXPERIMENTAL METHODS}

The measured detector in this work was fabricated in a relatively simple way. The Ti film was evaporated on a highresistivity silicon substrate and the micro-bridge was patterned using optical lithography enly. The photoresist patterning Ti structure was firstly coated on silicon substrate and a 37-nmthick Ti film was e-beam evaporated in an ultrahigh vacuum 
environment. Then the Ti structure was made by lift-off process. The $\mathrm{Nb}$ electrode was fabricated in a similar lift-off process. Before the deposition of $\mathrm{Nb}$ film, RF cleaning was used to remove the oxidation on the surface of Ti film. The detector's detector contact electrodes and outgoing leads were made of niobium $(\mathrm{Nb})$ with a $T_{\mathrm{c}}$ of around $9 \mathrm{~K}$. The schematic diagram and scanning electron microscope (SEM) photo of a detector's the detector central part is shown in Fig. 1. Note that the shown SEM photo was taken for a similar detector from the same batch since we want to avoid the risk of damage.

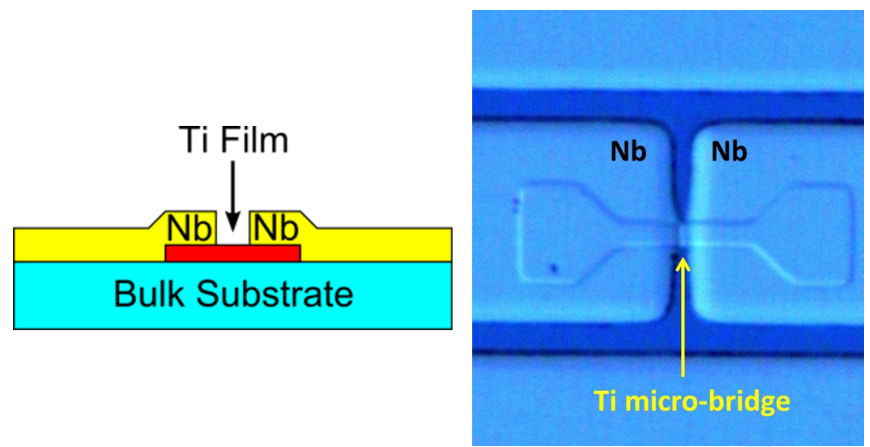

Fig. 1. Schematic diagram and SEM photo of the detector central part. The Ti film was evaporated on a high-resistivity silicon substrate and the micro-bridge was patterned using optical lithography enly.

The resistive transition ( $\mathrm{R}-\mathrm{T}$ curve) of the detector was measured by a sensitive AC resistance bridge. The detector was mount on the $300 \mathrm{mK}$ cold plate of an absorption refrigerator. The measured R-T curve of the detector is shown in Fig. 2. Clearly there are two transitions, with the high one at $434 \mathrm{mK}$ due to the $\mathrm{Nb} / \mathrm{Ti}$ contacts and the lower one at $412 \mathrm{mK}$ due to the $\mathrm{Ti}$ micro-bridge itself. Its normal-state resistance is approximately $17 \Omega$. The broadening of transition width might account for inhomogeneity of the Ti film. We use AFM (atomic force microseopy) to get surface proflle of a $\mathrm{Ti}$ film sample. The measured results show that the Ti fllm surface is quite rough. The surface profile of Ti film was measured by atomic force microscopy (AFM). The AFM results show that the height variation of Ti film surface is from $-2.0 \mathrm{~nm}$ to $2.0 \mathrm{~nm}$. The RMS roughness is around $0.6 \mathrm{~nm}$. The transition at different parts might be slightly different so that the transition of the whole sample might be broadened.

With respect to the measured normal-state resistance, we applied a constant current through a $0.68 \Omega$ metal-film resistor in parallel with the detector to realize a constant-voltage bias in the detector's operation. [23] [24] The biasing wires applying constant-current passed through home-made low pass RC filters to suppress common-mode and differential-mode electromagnetic interferences. In order to suppress commonmode and differential-mode electromagnetic interferences, a home-made low-pass filtering circuit was inserted in the biasing wires at the feed-though connector on the cryostat. The output current was read out by a low noise amplifier (a commercial DC SQUID, superconducting quantum interference device, amplifier). [24],[25] [25], [26] The DC SQUID chip was thermally anchored to the $2 \mathrm{~K}$ stage of the absorption refrigerator and its bias and control circuits were at room

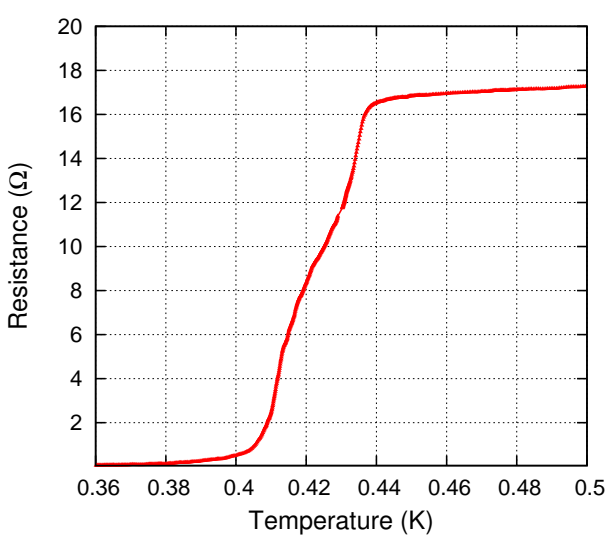

Fig. 2. R-T curve of the measured detector. There are two transitions, with the high one due to the $\mathrm{Nb} / \mathrm{Ti}$ contacts and the lower one due to the $\mathrm{Ti}$ micro-bridge itself. Its normal-state resistance is approximately $17 \Omega$.

temperature. The input coil of the DC SQUID chip was in series with the detector by a superconducting twisted pair to reduce the parasitic resistance. The setup with constant-voltage bias and current readout was used to study the electro-thermal properties and electrical noise performance.

We also measured the low frequency noise of a $\mathrm{Ti}$ film sample which is on the same wafer as the investigated detector to understand the film property. The current noise spectra was measured at the output of the SQUID amplifier by an FFT spectrum analyzer. By fitting the measured current noise spectra to empirical Hooge model [26],[27] [27], [28], the Hooge constant of this film is obtained.

For comparison purposes, a sample of $25 \mu \mathrm{m} \times 25 \mu \mathrm{m} \times$ $51 \mathrm{~nm}$ was fabricated and then tested in a setup introducing a laser source to get optical response.[22] [18] The measurement was done in a dilution refrigrator with another set of bias and DC SQUID amplifier. [28],[29] [29], [30] The transition temperature was measured $300 \mathrm{mK}$ and the normal-state resistance $8.3 \Omega$ using R-T measurement setup as above. A shunt resistor of $20 \mathrm{~m} \Omega$ was used to realize a constant-voltage bias. The detector was coupled to a single mode optical fiber which was connected to the light source at room temperature outside the cryostat. The laser source generates periodic pulses by a home-made laser at wavelength of $1550 \mathrm{~nm}$, a modulator, and a variable optical attenuator. The optical response was recorded by a digital osilloscope and then analyzed to get relaxation time.

\section{RESULTS AND DISCUSSION}

\section{A. Hooge constant of the Ti film}

The current noise spectra measured at the output of the SQUID amplifier by an FFT spectrum analyzer are shown in Fig. 3 for different bias voltages. The 1/f noise at low frequencies can be clearly observed. Using the empirical Hooge model [26],[27], we fitted the measured current noise spectra. The Hooge constant in this fitting was found to be $\alpha_{\mathrm{H}} \approx 2.64 \times 10^{-2} \pm 0.13 \times 10^{-2}$, which is greater than that suggested for pure metals $\left(\alpha_{\mathrm{H}} \approx 2 \times 10^{-3}\right)$. This is a strong indication of disorder existing in this Ti film. 
1

2

3

4

5

6

7

8

9

10

11

12

13

14

15

16

17

18

19

20

21

22

23

24

25

26

27

28

29

30

31

32

33

34

35

36

37

38

39

40

41

42

43

44

45

46

47

48

49

50

51

52

53

54

55

56

57

58

59

60

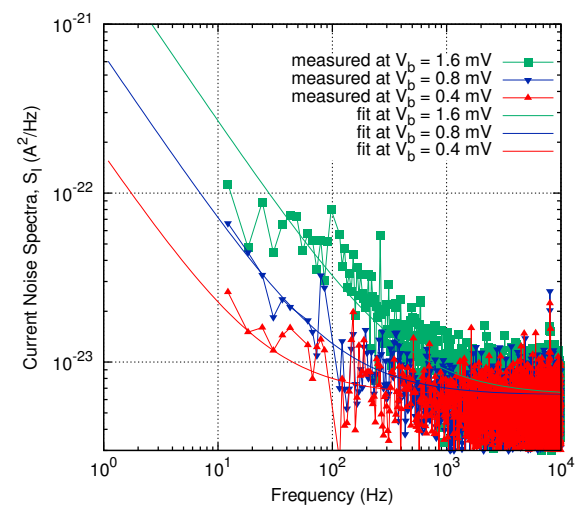

Fig. 3. Low-frequency current noise spectra of a Ti sample. The fitting is with Hooge model low frequency noise as well as Johnson noise and readout noise.

\section{B. Electro-thermal properties and electrical noise}

We investigated the detector characteristics including its thermal conductance, electro-thermal feedback loop gain, and NEP. They are all evaluated in terms of the detector's currentvoltage (I-V) curves at different temperatures. The normalstate resistance from the I-V curves is also approximately $17 \Omega$ in agreement with that from the R-T curve. The overall thermal conductance of a bolometric detector can be ealetlated extracted by the isothermal method [30] [31] from its measured I-V curves at different bath temperatures. The extracted overall thermal conductance $G_{\text {mea }} G_{\text {ext }}$ of our Ti bolometric detector was $170 \mathrm{pW} / \mathrm{K}-280 \mathrm{pW} / \mathrm{K}$ at $388 \mathrm{mK}$. Fig. 4 shows the measured I-V curves at temperature from $388 \mathrm{mK}$ to $404 \mathrm{mK}$ as well as the thermal conductance calculated from measured $\mathrm{I}-\mathrm{V}$ curves.

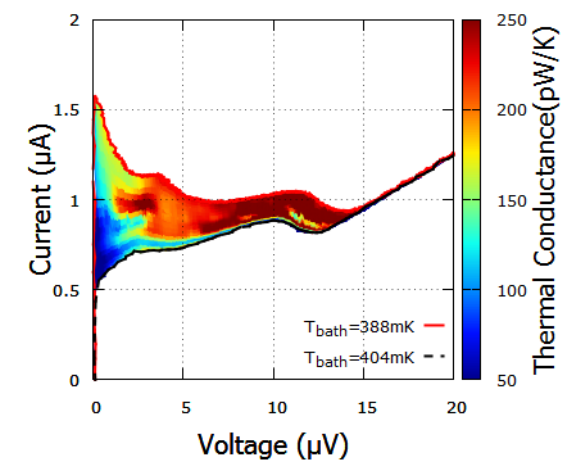

Fig. 4. I-V curves and thermal conductance calculated from them. The normalstate resistance from the I-V curves is in agreement with that from the $\mathrm{R}-\mathrm{T}$ curve. The thermal conductance of our $\mathrm{Ti}$ bolometric detector was $170 \mathrm{pW} / \mathrm{K}-280 \mathrm{pW} / \mathrm{K}$ at $388 \mathrm{mK}$.

The electro-thermal feedback (ETF) plays an important role in bolometric detector operation.\{31]-[33] [32]-[34] The feedback effect is quantified by the ETF loop gain $\mathscr{L}_{I}$, which can be expressed as $\mathscr{L}_{I}=\left(Z_{0}-R\right) /\left(Z_{0}+R\right)$, where $Z_{0}$ is the dynamic impedance $\left(\frac{\mathrm{d} V}{\mathrm{~d} I}\right)$. [32] [33] The ETF loop gain calculated with the measured I-V curve at $388 \mathrm{mK}$ is shown in Fig. 5. The ETF loop gain has two peaks along the increment of biasing voltage. The peak at high biasing voltage is higher than the one at low biasing voltage. The large loop gain leads to a constant Joule power dissipation in detector (the powervoltage (P-V) curve shown in Fig. 5).

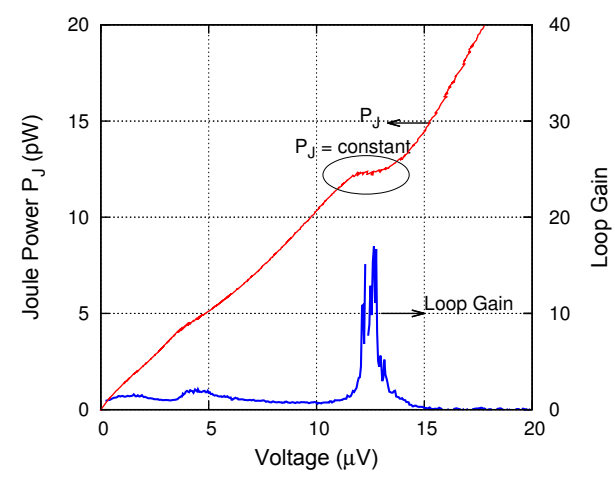

Fig. 5. Joule power and ETF loop gain. ETF loop gain has two peaks along the increment of biasing voltage. The larger one leads to a constant Joule power dissipation observed in the $\mathrm{P}-\mathrm{V}$ curve.

We characterized the NEP of the Ti bolometric detector investigated here, which is a frequently used figure of merit to characterize direct detectors. [2] The NEP is the square root of the power spectral density of the power-referred noise. The power-referred noise of a superconducting bolometric detector is indeed the sum of the contributions of different noises including the thermal fluctuation noise (TFN, or phonon noise), the Johnson noises from the detector resistance $R_{0}$ and load resistance $R_{\mathrm{L}}$, and the noise from the readout electronics (here the SQUID amplifier). It is frequency dependent and when the detector was operating at low frequency it can be calculated as [31] [32]

$$
\begin{aligned}
\mathrm{NEP}^{2} & =S_{P}=\frac{i_{\mathrm{n}}^{2}}{\left|\mathscr{R}_{I}\right|^{2}} \\
& =S_{P}^{\mathrm{TFN}}+S_{V}^{R_{0}} I_{0}^{2} \frac{1}{\mathscr{L}_{I}^{2}}+S_{V}^{R_{\mathrm{L}}} I_{0}^{2} \frac{\left(\mathscr{L}_{I}-1\right)^{2}}{\mathscr{L}_{I}^{2}}+\frac{S_{I}^{\mathrm{SQ}}}{\left|\mathscr{R}_{I}\right|^{2}},
\end{aligned}
$$

where $i_{\mathrm{n}}^{2}$ is the overall current noise spectral density, $\mathscr{R}_{I}$ the DC responsivity, $S_{P}^{\mathrm{TFN}}, S_{V}^{R_{0}}, S_{V}^{R_{\mathrm{L}}}$, and $S_{I}^{\mathrm{SQ}}$ the different equivalent noise power spectral density, $I_{0}$ the bias current. The DC responsivity can be calculated from the I-V curves as [32] [33]

$$
\mathscr{R}_{I}=-\frac{1}{V_{0}} \frac{Z_{0}-R_{0}}{2\left(Z_{0}+R_{\mathrm{L}}\right)} .
$$

At $388 \mathrm{mK}$, we measured the output noise spectral density $i_{\mathrm{n}}$ at $f=3 \mathrm{kHz}$ and calculated the measured NEP as $i_{\mathrm{n}} / \mathscr{R}_{I}$. NEP due to different contributions are also shown in Fig. 6 using equations as [31\} [32]

$$
\begin{gathered}
\mathrm{NEP}_{\mathrm{TFN}}=\sqrt{S_{P}^{\mathrm{TFN}}}=\sqrt{4 k_{\mathrm{B}} T_{\mathrm{c}}^{2} G}, \\
\mathrm{NEP}_{\mathrm{J}}=\sqrt{I_{0}^{2} \frac{4 k_{\mathrm{B}} T_{\mathrm{c}}}{R_{0}} \frac{1}{\mathscr{L}_{I}^{2}}+I_{0}^{2} \frac{4 k_{\mathrm{B}} T_{\mathrm{c}}}{R_{\mathrm{L}}} \frac{\left(\mathscr{L}_{I}-1\right)^{2}}{\mathscr{L}_{I}^{2}}}, \\
\mathrm{NEP}_{\mathrm{SQUID}}=\frac{i_{\mathrm{n}, \mathrm{SQUUD}}}{\mathscr{R}_{I}} \\
\mathrm{NEP}_{\text {total }}=\sqrt{S_{P}} .
\end{gathered}
$$


The measured NEP is slightly larger than the calculated total NEP. The NEP, when biased at points from $2.5 \mu \mathrm{V}$ to $13 \mu \mathrm{V}$, is around $10^{-16} \mathrm{~W} / \mathrm{Hz}^{0.5}$ which can fulfill the sensitivity requirement for ground-based astronomical application. The minimum measured NEP is approximately $5.9 \times 10^{-17} \mathrm{~W} / \mathrm{Hz}^{0.5}$ at a bias voltage of $3 \mu \mathrm{V}$ and $6.4 \times 10^{-17} \mathrm{~W} / \mathrm{Hz}^{0.5}$ at a bias voltage of $12 \mu \mathrm{V}$.

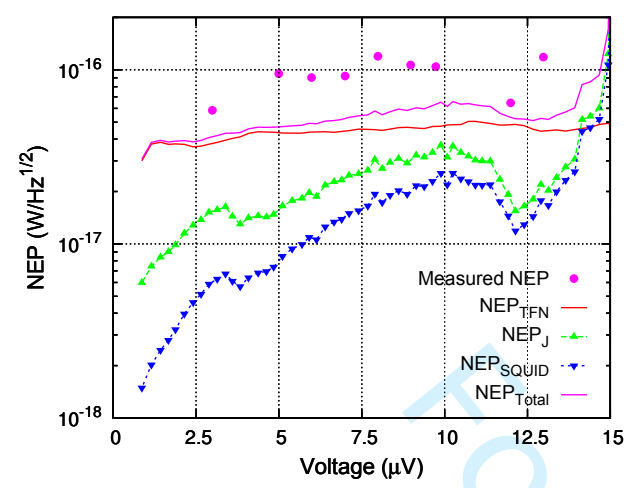

Fig. 6. Measured NEP and calculated different NEP contributions.

\section{Small heat capacity and small time constant}

The heat capacity $(C)$ of a thin normal metal film can be estimated calculated according to $C_{\mathrm{n}}=\gamma T V$, where $\gamma$ is the Sommerfeld constant, $V$ the device volume, while the phonon heat capacity is negligible at temperatures below $1 \mathrm{~K}$. For superconducting film at critical temperature the heat capacity is $2.43 C_{\mathrm{n}}$. The TES is operated within the superconducting transition so that $C$ varies in between $C_{\mathrm{n}}$ and $2.43 C_{\mathrm{n}}$. In our case $C=4.5 \times 10^{-18}-10.9 \times 10^{-18} \mathrm{JK}^{-1}$ (using $\gamma=310 \mathrm{Jm}^{-3} \mathrm{~K}^{-2}$ for bulk Ti [14]). A rough estimation of energy resolution [31] [32] using $\Delta E=\sqrt{4 k T_{0}^{2} C}$ was around $30-73 \mathrm{meV}$ (energy of around $3-7 \mathrm{THz}$ photon). This enables the applications in PNR detection of optical/IR photons.

The thermal time constant $\tau$ is $C / G$, where $C$ is the heat capacity of the detector, $G$ the thermal conductance. The estimated extracted time constant using $G_{\text {mea }} G_{\text {ext }}$ is thus $\tau_{\text {mea }}=C / G_{\text {mea }} \approx 19 \mathrm{~ns}-32 \mathrm{~ns} \tau_{\mathrm{ext}}=C / G_{\mathrm{ext}} \approx 19 \mathrm{~ns}-77 \mathrm{~ns}$. For a Ti TES detector the major part of thermal conductance is the one between the electrons and phonons (electron-phonon conductance $G_{\mathrm{e}-\mathrm{ph}}$ ). The electron-phonon conductance for metals within a small volume at low temperatures can be given by $G_{\mathrm{e}-\mathrm{ph}}=n \Sigma V T^{n-1}$ when the electron-phonon temperature difference $\left(\left|T_{\mathrm{e}}-T_{\mathrm{ph}}\right|\right)$ is much smaller than the phonon temperature $\left(T_{\mathrm{ph}}\right)$, where $\Sigma$ is a material-dependent constant, $V$ the volume.[31] [32] The index $n$ can vary between 4 and 6 . Some experiments suggest that $G_{\mathrm{e}-\mathrm{ph}}(T)=1.56 \times 10^{9} V T^{3}$ above $300 \mathrm{mK}$ for thin $\mathrm{Ti}$ films on $\mathrm{Si}$ substrate. $\{14],[34]$ [14], [35] With this suggestion of approximation, the electronphonon thermal conductance of our Ti bolometric detector is $G_{\mathrm{e}-\mathrm{ph}} \approx 3.4 \mathrm{pW} / \mathrm{K}$ and the electron-phonon relaxation time constant is therefore $\tau_{\mathrm{e} \text {-ph }}=C / G_{\mathrm{e} \text {-ph }} \approx 1.3 \mu \mathrm{s}$. The electronphonon thermal conductance $G_{\mathrm{e} \text {-ph }}$ is much smaller than the extracted overall thermal conductance $G_{\text {mea }} G_{\text {ext }}$ evaluated from measured I-V curves. This implies that there might be an extra thermal link besides electron-phonon cooling. A diffusion cooling path through electrodes might account for that.

The diffusion cooling time constant due to diffusion cooling path can be estimated calculated according to $\tau_{\text {diff }}=$ $L^{2} /\left(\pi^{2} D\right)$, where $L$ is the micro-bridge length and $D$ the electron diffusion constant.[19],[20] [21], [22] Assuming the electron diffusion constant $D=1 \mathrm{~cm}^{2} / \mathrm{s}$ [21] and a 1$\mu \mathrm{m}$ long micro-bridge, we have an estimated diffusion time $\tau_{\text {diff }} \approx 1.0 \mathrm{~ns}$. Note that the total relaxation rate $1 / \tau=$ $1 / \tau_{\text {e-ph }}+1 / \tau_{\text {diff. }}$. If $\tau_{\text {e-ph }} \gg \tau_{\text {diff }}$, we get $\tau \approx \tau_{\text {diff. }}$. However as can be seen for our detector, the $\tau_{\text {mea }} \approx 19 \mathrm{~ns}-32 \mathrm{~ns} \tau_{\text {ext }}$ is in between the electron-phonon one $\tau_{\mathrm{e} \text {-ph }} \approx 1.3 \mu$ s and diffusion cooling one $\tau_{\text {diff }} \approx 1.0 \mathrm{~ns}$. It implies that the diffusion cooling is weakened to a certain degree (this leads to a greater time constant) but not well enough suppressed (if well suppressed, $\tau$ should be close to $\tau_{\mathrm{e}-\mathrm{ph}}$ ). In our detector, the area of the interface between the Ti micro-bridge and $\mathrm{Nb}$ electrodes is larger than the micro-bridge and the R-T curve shows that the transition temperature of contact area is not high enough to suppress the diffusion of electrons. For comparison, a sample of $25 \mu \mathrm{m} \times 25 \mu \mathrm{m} \times 51 \mathrm{~nm}$ with contact electrode area of $25 \mu \mathrm{m} \times 2 \mu \mathrm{m}$ was fabricated and tested by recording its optical response to obtain its actual relaxation time constant.

The optical response output $\delta V(t)$ of TES to input pulse can be fitted to formula as $\{22]$ [18]

$$
\delta V(t)=A\left[\exp \left(-\frac{t-t_{0}}{\tau_{\text {decay }}}\right)-\exp \left(-\frac{t-t_{0}}{\tau_{\text {rise }}}\right)\right],
$$

where $A$ is a constant of pulse height, $t_{0}$ the incident time, $\tau_{\text {rise }}$ the rise time constant, and $\tau_{\text {decay }}$ the relaxation time constant. Averaged pulse response was recorded and fitted using the above equations and the fitting gives $\tau_{\text {rise }}=0.12 \mu \mathrm{s}$ and $\tau_{\text {decay }}=0.93 \mu$ s. The heat capacity of the large area detector is $C_{1}=3.0 \mathrm{fJ} / \mathrm{K}$ (using $\gamma T V$ ). The electron-phonon thermal conductance is $G_{\mathrm{e}-\mathrm{ph}, \mathrm{l}}=1.34 \mathrm{nW} / \mathrm{K}$ (using $1.56 \times 10^{9} V T^{3}$ ) and thus electron-phonon thermal time constant is $\tau_{\mathrm{e}-\mathrm{ph}}=$ $2.2 \mu$ s. Due to ETF effect the actual relaxation time constant is smaller than electron-phonon thermal time constant, [33] [34] but still it can be seen that they are in the same order for this large area sample. This result provides a comparison to that of our small-size Ti detector. The time constant of the small-area detector was smaller than that of the larger one.

\section{CONCLusions}

In conclusion, we have fabricated a small-size Ti TES detector and characterized its electrical and thermal properties. The minimum measured electrical NEP is around $6 \times 10^{-17} \mathrm{~W} / \mathrm{Hz}^{0.5}$, which is suitable for ground-based astronomical observations. The R-T curve, the measured thermal conductance, and the thermal time constant indicate that the diffusion cooling contributes an important part in the thermal relaxation process. With high sensitivity, fast response, and low heat capacity, the small-size Ti TES detector is suitable for power detection as well as for PNR detection in astronomical applications. 


\section{ACKNOWLEDGMENT}

We thank Joseph MARTINO, Damien PRELE, Fabrice VOISIN, and Michel PIAT of Laboratoire AstroParticule et Cosmologie (APC, Univ. Paris-7, France) for help with the I-V testing setup. We thank Yang LIU and Qiang ZHANG of University of Science and Technology of China (USTC, China) for help with the optical response testing setup.

\section{REFERENCES}

[1] R. C. Jones, "The General Theory of Bolometer Performance," Journal of the Optical Society of America, vol. 43, no. 1, pp. 1-10, 1953.

[2] P. L. Richards, "Bolometers for infrared and millimeter waves," Journal of Applied Physics, vol. 76, no. 1, pp. 1-24, Jul. 1994.

[3] D. McCammon, "Thermal Equilibrium Calorimeters An Introduction," in Cryogenic Particle Detection, ser. Topics in Applied Physics, C. Enss, Ed. Berlin Heidelberg: Springer-Verlag, 2005, vol. 99, pp. 1-34.

[4] J. M. Gildemeister, A. T. Lee, and P. L. Richards, "A fully lithographed voltage-biased superconducting spiderweb bolometer," Applied Physics Letters, vol. 74, no. 6, pp. 868-870, Feb. 1999.

[5] C. L. Kuo, J. J. Bock, J. A. Bonetti, J. Brevik, G. Chattopadhyay, P. K. Day, S. Golwala, M. Kenyon, A. E. Lange, H. G. LeDuc, H. Nguyen, R. W. Ogburn, A. Orlando, A. Transgrud, A. Turner, G. Wang, and J. Zmuidzinas, "Antenna-coupled TES bolometer arrays for CMB polarimetry,” Proc. SPIE, vol. 7020, p. 70201I, 2008.

[6] B. Cabrera, R. M. Clarke, P. Colling, A. J. Miller, S. Nam, and R. W. Romani, "Detection of single infrared, optical, and ultraviolet photons using superconducting transition edge sensors," Applied Physics Letters, vol. 73, no. 6, pp. 735-737, Aug. 1998.

[7] R. W. Romani, T. J. Bay, J. Burney, and B. Cabrera, "Transition Edge Cameras for Fast Optical Spectrophotometry," in High Time Resolution Astrophysics, ser. Astrophysics and Space Science Library, D. Phelan, O. Ryan, and A. Shearer, Eds. Netherlands: Springer, 2008, vol. 351, pp. 311-325.

[8] K. D. Irwin, G. C. Hilton, D. A. Wollman, and J. M. Martinis, "X-ray detection using a superconducting transitionedge sensor microcalorimeter with electrothermal feedback," Applied Physics Letters, vol. 69, no. 13, pp. 1945-1947, Sep. 1996.

[9] J. N. Ullom and D. A. Bennett, "Review of superconducting transition-edge sensors for X-ray and gamma-ray spectroscopy," Superconductor Science and Technology, vol. 28, no. 8, p. 084003, 2015.

[10] J. E. Austermann, K. A. Aird, and et al., "SPTpol: an instrument for CMB polarization measurements with the South Pole Telescope," Proc. SPIE, vol. 8452, p. 84521E, 2012.

[11] W. S. Holland, D. Bintley, E. L. Chapin, A. Chrysostomou, G. R. Davis, J. T. Dempsey, W. D. Duncan, M. Fich, P. Friberg, M. Halpern, K. D. Irwin, T. Jenness, B. D. Kelly, M. J. MacIntosh, E. I. Robson, D. Scott, P. a. R. Ade, E. Atad-Ettedgui, D. S. Berry, S. C. Craig, X. Gao, A. G. Gibb, G. C. Hilton, M. I. Hollister, J. B. Kycia, D. W. Lunney, H. McGregor, D. Montgomery, W. Parkes, R. P. J. Tilanus, J. N. Ullom, C. A. Walther, A. J. Walton, A. L. Woodcraft, M. Amiri, D. Atkinson, B. Burger, T. Chuter, I. M. Coulson, W. B. Doriese, C. Dunare, F. Economou, M. D. Niemack, H. a. L. Parsons, C. D. Reintsema, B. Sibthorpe, I. Smail, R. Sudiwala, and H. S. Thomas, "SCUBA-2: the 10000 pixel bolometer camera on the James Clerk Maxwell Telescope," Monthly Notices of the Royal Astronomical Society, vol. 430, no. 4, pp. 2513-2533, Apr. 2013.

[12] B. S. Karasik, B. Delaet, W. R. McGrath, J. Wei, M. E. Gershenson, and A. V. Sergeev, "Experimental study of superconducting hotelectron sensors for submm astronomy," IEEE Transactions on Applied Superconductivity, vol. 13, no. 2, pp. 188-191, 2003.

[13] B. S. Karasik, D. Olaya, J. Wei, S. Pereverzev, M. E. Gershenson, J. H. Kawamura, W. R. McGrath, and A. V. Sergeev, "Record-Low NEP in Hot-Electron Titanium Nanobolometers," IEEE Transactions on Applied Superconductivity, vol. 17, no. 2, pp. 293-297, Jun. 2007.

[14] B. S. Karasik, S. V. Pereverzev, D. Olaya, J. Wei, M. E. Gershenson, and A. V. Sergeev, "Noise Measurements in Hot-Electron Titanium Nanobolometers," IEEE Transactions on Applied Superconductivity, vol. 19, no. 3, pp. 532-535, Jun. 2009.

[15] B. S. Karasik, A. V. Sergeev, and D. E. Prober, "Nanobolometers for $\mathrm{THz}$ Photon Detection," IEEE Transactions on Terahertz Science and Technology, vol. 1, no. 1, pp. $97-111$, Sep. 2011.
[16] B. S. Karasik and A. V. Sergeev, "THz hot-electron photon counter," IEEE Transactions on Applied Superconductivity, vol. 15, no. 2, pp. 618-621, Jun. 2005.

[17] D. F. Santavicca, B. Reulet, B. S. Karasik, S. V. Pereverzev, D. Olaya, M. E. Gershenson, L. Frunzio, and D. E. Prober, "Energy resolution of terahertz single-photon-sensitive bolometric detectors," Applied Physics Letters, vol. 96, no. 8, pp. 083 505-083 505-3, Feb. 2010.

[18] D. Fukuda, G. Fujii, A. Yoshizawa, H. Tsuchida, R. Damayanthi, H. Takahashi, S. Inoue, and M. Ohkubo, "High Speed Photon Number Resolving Detector with Titanium Transition Edge Sensor," Journal of Low Temperature Physics, vol. 151, no. 1, pp. 100-105, 2008.

[19] C. Portesi, E. Taralli, L. Lolli, M. Rajteri, and E. Monticone, "Fabrication and characterization of fast TESs with small area for single photon counting," IEEE Transactions on Applied Superconductivity, vol. 25, no. 3, pp. 1-4, Jun. 2015.

[20] W. Zhang, J. Q. Zhong, W. Miao, Z. Wang, D. Liu, Q. J. Yao, S. C. Shi, T. J. Chen, and M. J. Wang, "Electrical Characteristics of Superconducting Ti Transition Edge Sensors," Journal of Low Temperature Physics, vol. 184, pp. 11-16, Oct. 2016

[21] B. S. Karasik, K. S. Il'in, E. V. Pechen, and S. I. Krasnosvobodtsev, "Diffusion cooling mechanism in a hotelectron NbC microbolometer mixer," Applied Physics Letters, vol. 68, no. 16, pp. 2285-2287, Apr. 1996.

[22] A. Skalare, W. R. McGrath, B. Bumble, H. G. LeDuc, P. J. Burke, A. A. Verheijen, R. J. Schoelkopf, and D. E. Prober, "Large bandwidth and low noise in a diffusioncooled hotelectron bolometer mixer," Applied Physics Letters, vol. 68, no. 11, pp. 1558-1560, Mar. 1996.

[23] L. Lolli, E. Taralli, M. Rajteri, T. Numata, and D. Fukuda, "Characterization of Optical Fast Transition-Edge Sensors With Optimized Fiber Coupling," IEEE Transactions on Applied Superconductivity, vol. 23, no. 3, pp. $2100904-2100904$, Jun. 2013.

[24] K. Irwin, S. Nam, B. Cabrera, B. Chugg, G. Park, R. P. Welty, and J. Martinis, "A self-biasing cryogenic particle detector utilizing electrothermal feedback and a SQUID readout," IEEE Transactions on Applied Superconductivity, vol. 5, no. 2, pp. 2690-2693, 1995.

[25] R. Cantor, L. P. Lee, A. Matlashov, and V. Vinetskiy, "A low-noise, twostage DC SQUID amplifier with high bandwidth and dynamic range," IEEE Transactions on Applied Superconductivity, vol. 7, no. 2, pp. 3033-3036, Jun. 1997.

[26] "pcSQUID TM advanced PC-based SQUID control electronics of STAR Cryoelectronics," http://www.starcryo.com/pcsquid/.

[27] F. N. Hooge, "1/f noise sources," IEEE Transactions on Electron Devices, vol. 41, no. 11, pp. 1926-1935, Nov 1994.

[28] G. P. Zhigal'skii, "1/ f noise and nonlinear effects in thin metal films," Physics-Uspekhi, vol. 40, no. 6, p. 599, 1997.

[29] D. Drung, C. Hinnrichs, and H.-J. Barthelmess, "Lownoise ultra-high-speed dc SQUID readout electronics," Superconductor Science and Technology, vol. 19, no. 5, p. S235, May 2006.

[30] "SQUID systems of Magnicon GmbH," http://www.magnicon.com/squid-systems/.

[31] H. Ekström, B. S. Karasik, E. L. Kollberg, and K. S. Yngvesson, "Conversion gain and noise of niobium superconducting hot-electronmixers," IEEE Transactions on Microwave Theory and Techniques, vol. 43, no. 4, pp. $938-947,1995$.

[32] K. D. Irwin and G. C. Hilton, "Transition-Edge Sensors," in Cryogenic Particle Detection, ser. Topics in Applied Physics, C. Enss, Ed. Berlin Heidelberg: Springer-Verlag, Jan. 2005, vol. 99, pp. 63-150.

[33] J. C. Mather, "Bolometer noise: nonequilibrium theory," Applied Optics, vol. 21, no. 6, pp. 1125-1129, Mar. 1982.

[34] K. D. Irwin, "An application of electrothermal feedback for high resolution cryogenic particle detection," Applied Physics Letters, vol. 66, pp. 1998-2000, Apr. 1995.

[35] J. Wei, D. Olaya, B. S. Karasik, S. V. Pereverzev, A. V. Sergeev, and M. E. Gershenson, "Ultrasensitive hot-electron nanobolometers for terahertz astrophysics," Nature Nanotechnology, vol. 3, no. 8, pp. 496500, 2008. 


\title{
Fast-response Superconducting Titanium Bolometric Detectors
}

\author{
JiaQiang Zhong, Wen Zhang, Wei Miao, Dong Liu, Zheng Wang, Wen-Ying Duan, Feng Wu, Kun Zhang, \\ Qijun Yao, Sheng-cai Shi, Ming-Jye Wang, and François Pajot
}

\begin{abstract}
High time resolution optical/Infrared (IR) astronomy will require fast response detectors. In order to meet such requirement, cryogenic optical/IR detectors, such as superconducting tunnel junctions (STJ) and transition-edge sensors (TES), are under development. Of these cryogenic detectors, superconducting titanium (Ti) bolometric detectors, with a transition temperature of around $300 \mathrm{mK}$, based on the electron-phonon decoupling mechanism, is promising in fast response. In this paper, we report on the characterization of electro-thermal properties of a small-size $(1 \mu \mathrm{m} \times 1 \mu \mathrm{m} \times 37 \mathrm{~nm})$ superconducting Ti detector. The low frequency noise of Ti film was fitted by empirical Hooge model. A discrepancy between the Hooge constant of the Ti film and that of normal metals shows disorder in the $\mathrm{Ti}$ film. Thermal conductance was extracted via measured currentvoltage curves at different bath temperatures. The time constant was calculated as tens of nanoseconds, which was faster than expected. The fast response potentially enables the high speed optical/IR astronomical observations. Detailed experimental and analytical results are presented.
\end{abstract}

Index Terms-Superconducting detectors, bolometers, electrothermal feedback, noise equivalent power.

\section{INTRODUCTION}

B OLOMETRIC detectors based on weak thermal coupling between an absorber and a heat sink have been widely used in radiation detection. [1]-[3] Among them, superconducting bolometric detectors (a.k.a. TES, transitionedge sensors) have been employed in a large wavelengthrange, from millimeter wave, optical and infrared (IR), to X-rays. [4]-[9] TES detectors with very high sensitivity and relatively large arrays are playing an increasingly important role in millimeter and sub-millimeter astronomy. [10], [11]

This work was supported in part by National Key R\&D program of China under Grant 2017YFA0304003, NSFC under Grants U1731119, 11673073 , U1831202, 11473075, Chinese Academy of Sciences (CAS) Key Research Program of Frontier Sciences under Grant QYZDJ-SSW-SLH043, CAS Key Technology Research Team Program under Grant GJJSTD20180003, and by the CAS joint Key Lab for Radio Astronomy.(Corresponding author: Shengcai Shi, e-mail: scshi@pmo.ac.cn.)

JiaQiang Zhong, Wen Zhang, Wei Miao, Dong Liu, Zheng Wang, Wen-Ying Duan, Feng Wu, Kun Zhang, QiJun Yao and Sheng-cai Shi are with the Purple Mountain Observatory and Key Laboratory of Radio Astronomy, Chinese Academy of Sciences, Nanjing 210034, China.(email: jqzhong@pmo.ac.cn; wzhang@pmo.ac.cn; wmiao@pmo.ac.cn; dliu@pmo.ac.cn; $\quad$ zhwang@pmo.ac.cn; wyduan@pmo.ac.cn; wufeng@pmo.ac.cn; zhangkun@pmo.ac.cn; yqj@mwlab.pmo.ac.cn; scshi@pmo.ac.cn.)

Ming-Jye Wang is with Institute of Astronomy and Astrophysics, Academia Sinica, Taipei, Taiwan.(e-mail: mingjye@asiaa.sinica.edu.tw)

François Pajot is with Institut de Recherche en Astrophysique et Planétologie, Toulouse, France.(e-mail: francois.pajot@irap.omp.eu)

Color versions of one or more of the figures in this paper are available online at http://ieeexplore.iee.org.

Digital Object Identifier will be inserted here upon acceptance.
For example, the instrument SCUBA-2 on the James Clerk Maxwell Telescope (JCMT) has 10,000 TES and employs time domain multiplexing readout. [11] In ground-based astronomical applications, the requirement of detector sensitivity, i.e., noise equivalent power (NEP), is background-limited at the level of $10^{-17} \mathrm{~W} / \mathrm{Hz}^{0.5}-10^{-16} \mathrm{~W} / \mathrm{Hz}^{0.5}$ at millimeter wavelength and for future space telescopes at the level of $\sim 10^{-20} \mathrm{~W} / \mathrm{Hz}^{0.5}$. [12]-[15] The key to achieve such high sensitivity for TES is small heat capacity. The small heat capacity is obtained by minimizing detector pixel size. [12], [15] At the same time, by using high critical temperature materials such as $\mathrm{Ti}$, fast response photon-number-resolving detection for terahertz/far infrared (THz/FIR) might be possible. [15][17] By coupling to single-mode optical/IR fiber, it is also suitable for optical/IR detection. [18], [19]

Headed for high sensitivity and fast response, we developed small-area Ti TES. [20] In the current paper, we report on the characterization of a Ti TES detector with a micro-bridge of $1 \mu \mathrm{m} \times 1 \mu \mathrm{m} \times 37 \mathrm{~nm}$. The characteristics such as resistive transition, thermal conductance, electro-thermal feedback loop gain, and noise were studied. The time constant was evaluated using the heat capacity and the thermal conductance. The most important result is that the extracted response time constant is shorter than the expected electron-phonon relaxation time constant. This result indicates that the overall thermal conductance was higher than the electron-phonon one due to diffusion cooling via contacts. [21], [22] The diffusion cooling via contacts takes effect in the small-size detector whereas it is negligible in a large-area detector. A Ti TES detector with relatively large size of $25 \mu \mathrm{m} \times 25 \mu \mathrm{m} \times 51 \mathrm{~nm}$ was then fabricated and tested coupled to a laser source. The optical response using large-area detector was recorded and analyzed to get relaxation time. [18], [23] The time constant of the small-size detector was shorter than that of the larger one. With high sensitivity, fast response, and low heat capacity, the small-size Ti TES detector is promising for power detection as well as for PNR detection in astronomical applications.

\section{EXPERIMENTAL METHODS}

The measured detector in this work was fabricated in a relatively simple way. The Ti film was evaporated on a high-resistivity silicon substrate and the micro-bridge was patterned using optical lithography The photoresist patterning Ti structure was firstly coated on silicon substrate and a 37-nm-thick Ti film was e-beam evaporated in an ultrahigh vacuum environment. Then the Ti structure was made by lift- 
off process. The $\mathrm{Nb}$ electrode was fabricated in a similar liftoff process. Before the deposition of Nb film, RF cleaning was used to remove the oxidation on the surface of Ti film. The detector contact electrodes and outgoing leads were made of niobium $(\mathrm{Nb})$ with a $T_{\mathrm{c}}$ of around $9 \mathrm{~K}$. The schematic diagram and scanning electron microscope (SEM) photo of the detector central part is shown in Fig. 1. Note that the shown SEM photo was taken for a similar detector from the same batch since we want to avoid the risk of damage.

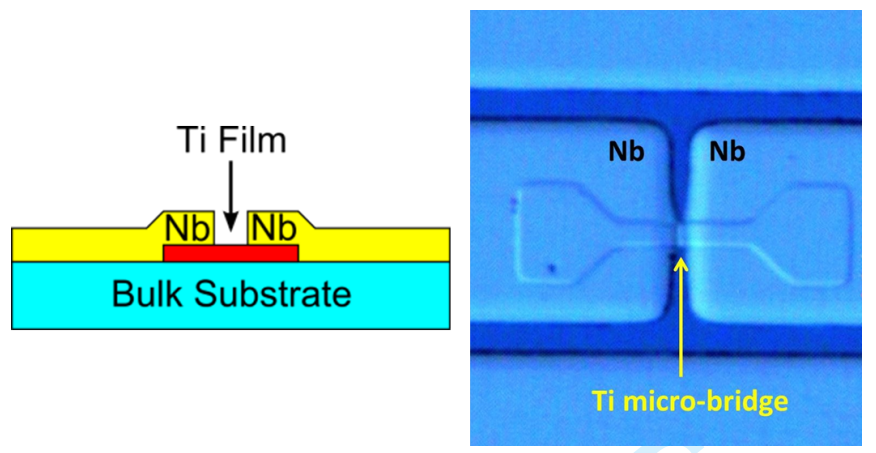

Fig. 1. Schematic diagram and SEM photo of the detector central part. The Ti film was evaporated on a high-resistivity silicon substrate and the microbridge was patterned using optical lithography.

The resistive transition (R-T curve) of the detector was measured by a sensitive AC resistance bridge. The detector was mount on the $300 \mathrm{mK}$ cold plate of an absorption refrigerator. The measured R-T curve of the detector is shown in Fig. 2. Clearly there are two transitions, with the high one at $434 \mathrm{mK}$ due to the $\mathrm{Nb} / \mathrm{Ti}$ contacts and the lower one at $412 \mathrm{mK}$ due to the $\mathrm{Ti}$ micro-bridge itself. Its normal-state resistance is approximately $17 \Omega$. The broadening of transition width might account for inhomogeneity of the Ti film. The surface profile of Ti film was measured by atomic force microscopy (AFM). The AFM results show that the height variation of Ti film surface is from $-2.0 \mathrm{~nm}$ to $2.0 \mathrm{~nm}$. The RMS roughness is around $0.6 \mathrm{~nm}$. The transition at different parts might be slightly different so that the transition of the whole sample might be broadened.

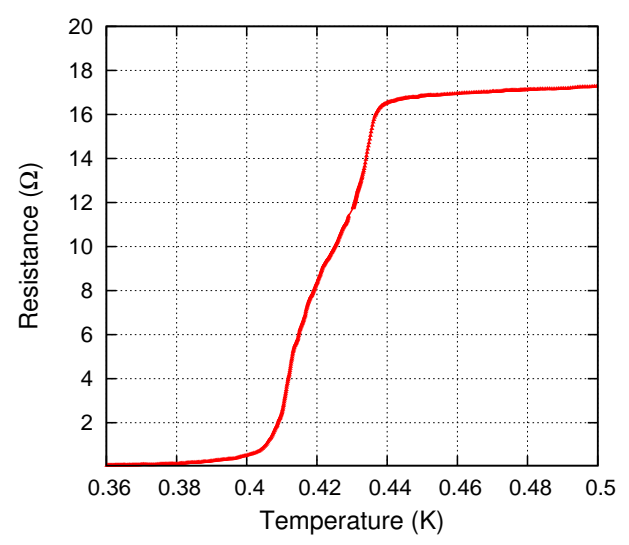

Fig. 2. R-T curve of the measured detector. There are two transitions, with the high one due to the $\mathrm{Nb} / \mathrm{Ti}$ contacts and the lower one due to the $\mathrm{Ti}$ micro-bridge itself. Its normal-state resistance is approximately $17 \Omega$.
With respect to the measured normal-state resistance, we applied a constant current through a $0.68 \Omega$ metal-film resistor in parallel with the detector to realize a constant-voltage bias. [24] In order to suppress common-mode and differential-mode electromagnetic interferences, a home-made low-pass filtering circuit was inserted in the biasing wires at the feed-though connector on the cryostat. The output current was read out by a low noise amplifier (a commercial DC SQUID, superconducting quantum interference device, amplifier). [25], [26] The DC SQUID chip was thermally anchored to the $2 \mathrm{~K}$ stage of the absorption refrigerator and its bias and control circuits were at room temperature. The input coil of the DC SQUID chip was in series with the detector by a superconducting twisted pair to reduce the parasitic resistance. The setup with constant-voltage bias and current readout was used to study the electro-thermal properties and electrical noise performance.

We also measured the low frequency noise of a Ti film sample which is on the same wafer as the investigated detector to understand the film property. The current noise spectra was measured at the output of the SQUID amplifier by an FFT spectrum analyzer. By fitting the measured current noise spectra to empirical Hooge model [27], [28], the Hooge constant of this film is obtained.

For comparison purposes, a sample of $25 \mu \mathrm{m} \times 25 \mu \mathrm{m} \times$ $51 \mathrm{~nm}$ was fabricated and then tested in a setup introducing a laser source to get optical response. [18] The measurement was done in a dilution refrigrator with another set of bias and DC SQUID amplifier. [29], [30] The transition temperature was measured $300 \mathrm{mK}$ and the normal-state resistance $8.3 \Omega$ using R-T measurement setup as above. A shunt resistor of $20 \mathrm{~m} \Omega$ was used to realize a constant-voltage bias. The detector was coupled to a single mode optical fiber which was connected to the light source at room temperature outside the cryostat. The laser source generates periodic pulses by a home-made laser at wavelength of $1550 \mathrm{~nm}$, a modulator, and a variable optical attenuator. The optical response was recorded by a digital osilloscope and then analyzed to get relaxation time.

\section{RESULTS AND DISCUSSION}

\section{A. Hooge constant of the Ti film}

The current noise spectra measured at the output of the SQUID amplifier by an FFT spectrum analyzer are shown in Fig. 3 for different bias voltages. The 1/f noise at low frequencies can be clearly observed. Using the empirical Hooge model, we fitted the measured current noise spectra. The Hooge constant in this fitting was found to be $\alpha_{\mathrm{H}} \approx 2.64 \times 10^{-2} \pm 0.13 \times 10^{-2}$, which is greater than that suggested for pure metals $\left(\alpha_{\mathrm{H}} \approx 2 \times 10^{-3}\right)$. This is a strong indication of disorder existing in this $\mathrm{Ti}$ film.

\section{B. Electro-thermal properties and electrical noise}

We investigated the detector characteristics including its thermal conductance, electro-thermal feedback loop gain, and NEP. They are all evaluated in terms of the detector's currentvoltage (I-V) curves at different temperatures. The normalstate resistance from the I-V curves is also approximately $17 \Omega$ in agreement with that from the R-T curve. The overall 


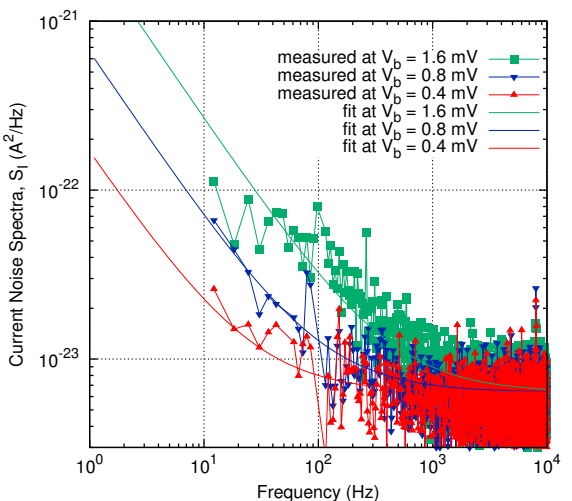

Fig. 3. Low-frequency current noise spectra of a Ti sample. The fitting is with Hooge model low frequency noise as well as Johnson noise and readout noise.

thermal conductance of a bolometric detector can be extracted by the isothermal method [31] from its measured I$\mathrm{V}$ curves at different bath temperatures. The extracted overall thermal conductance $G_{\text {ext }}$ of our Ti bolometric detector was $170 \mathrm{pW} / \mathrm{K}-280 \mathrm{pW} / \mathrm{K}$ at $388 \mathrm{mK}$. Fig. 4 shows the measured I-V curves at temperature from $388 \mathrm{mK}$ to $404 \mathrm{mK}$ as well as the thermal conductance calculated from measured I-V curves.

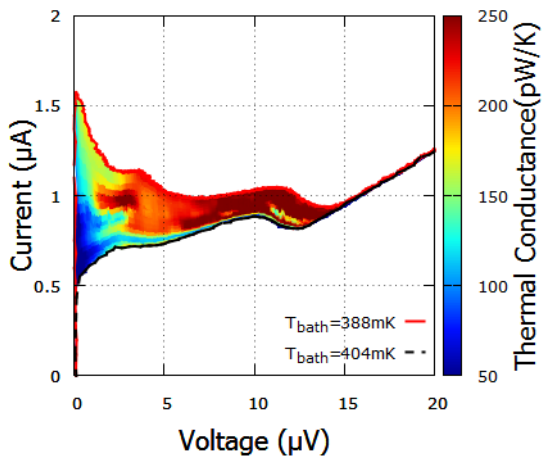

Fig. 4. I-V curves and thermal conductance calculated from them. The normalstate resistance from the $\mathrm{I}-\mathrm{V}$ curves is in agreement with that from the $\mathrm{R}-\mathrm{T}$ curve. The thermal conductance of our $\mathrm{Ti}$ bolometric detector was $170 \mathrm{pW} / \mathrm{K}-280 \mathrm{pW} / \mathrm{K}$ at $388 \mathrm{mK}$.

The electro-thermal feedback (ETF) plays an important role in bolometric detector operation. [32]-[34] The feedback effect is quantified by the ETF loop gain $\mathscr{L}_{I}$, which can be expressed as $\mathscr{L}_{I}=\left(Z_{0}-R\right) /\left(Z_{0}+R\right)$, where $Z_{0}$ is the dynamic impedance $\left(\frac{\mathrm{d} V}{\mathrm{~d} I}\right)$. [33] The ETF loop gain calculated with the measured I-V curve at $388 \mathrm{mK}$ is shown in Fig. 5. The ETF loop gain has two peaks along the increment of biasing voltage. The peak at high biasing voltage is higher than the one at low biasing voltage. The large loop gain leads to a constant Joule power dissipation in detector (the power-voltage (P-V) curve shown in Fig. 5).

We characterized the NEP of the Ti bolometric detector investigated here, which is a frequently used figure of merit to characterize direct detectors. [2] The NEP is the square root of the power spectral density of the power-referred noise. The power-referred noise of a superconducting bolometric

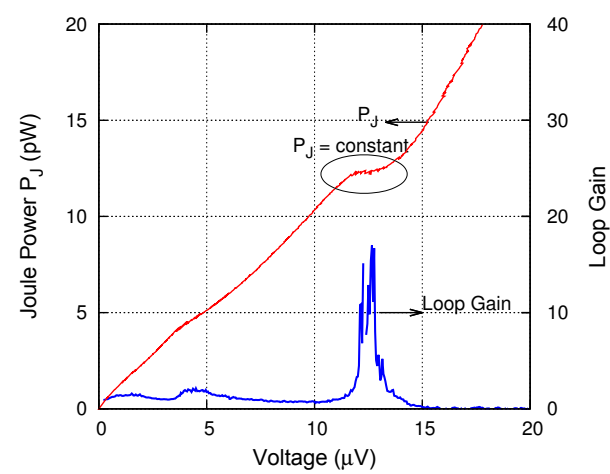

Fig. 5. Joule power and ETF loop gain. ETF loop gain has two peaks along the increment of biasing voltage. The larger one leads to a constant Joule power dissipation observed in the P-V curve.

detector is indeed the sum of the contributions of different noises including the thermal fluctuation noise (TFN, or phonon noise), the Johnson noises from the detector resistance $R_{0}$ and load resistance $R_{\mathrm{L}}$, and the noise from the readout electronics (here the SQUID amplifier). It is frequency dependent and when the detector was operating at low frequency it can be calculated as [32]

$$
\begin{aligned}
\mathrm{NEP}^{2} & =S_{P}=\frac{i_{\mathrm{n}}^{2}}{\left|\mathscr{R}_{I}\right|^{2}} \\
& =S_{P}^{\mathrm{TFN}}+S_{V}^{R_{0}} I_{0}^{2} \frac{1}{\mathscr{L}_{I}^{2}}+S_{V}^{R_{\mathrm{L}}} I_{0}^{2} \frac{\left(\mathscr{L}_{I}-1\right)^{2}}{\mathscr{L}_{I}^{2}}+\frac{S_{I}^{\mathrm{SQ}}}{\left|\mathscr{R}_{I}\right|^{2}},
\end{aligned}
$$

where $i_{\mathrm{n}}^{2}$ is the overall current noise spectral density, $\mathscr{R}_{I}$ the DC responsivity, $S_{P}^{\mathrm{TFN}}, S_{V}^{R_{0}}, S_{V}^{R_{\mathrm{L}}}$, and $S_{I}^{\mathrm{SQ}}$ the different equivalent noise power spectral density, $I_{0}$ the bias current. The DC responsivity can be calculated from the I-V curves as [33]

$$
\mathscr{R}_{I}=-\frac{1}{V_{0}} \frac{Z_{0}-R_{0}}{2\left(Z_{0}+R_{\mathrm{L}}\right)} .
$$

At $388 \mathrm{mK}$, we measured the output noise spectral density $i_{\mathrm{n}}$ at $f=3 \mathrm{kHz}$ and calculated the measured NEP as $i_{\mathrm{n}} / \mathscr{R}_{I}$. NEP due to different contributions are also shown in Fig. 6 using equations as [32]

$$
\begin{gathered}
\mathrm{NEP}_{\mathrm{TFN}}=\sqrt{S_{P}^{\mathrm{TFN}}}=\sqrt{4 k_{\mathrm{B}} T_{\mathrm{c}}^{2} G}, \\
\mathrm{NEP}_{\mathrm{J}}=\sqrt{I_{0}^{2} \frac{4 k_{\mathrm{B}} T_{\mathrm{c}}}{R_{0}} \frac{1}{\mathscr{L}_{I}^{2}}+I_{0}^{2} \frac{4 k_{\mathrm{B}} T_{\mathrm{c}}}{R_{\mathrm{L}}} \frac{\left(\mathscr{L}_{I}-1\right)^{2}}{\mathscr{L}_{I}^{2}}} \\
\mathrm{NEP}_{\mathrm{SQUID}}=\frac{i_{\mathrm{n}, \mathrm{SQUID}}}{\mathscr{R}_{I}} \\
\mathrm{NEP}_{\text {total }}=\sqrt{S_{P}} .
\end{gathered}
$$

The measured NEP is slightly larger than the calculated total NEP. The NEP, when biased at points from $2.5 \mu \mathrm{V}$ to $13 \mu \mathrm{V}$, is around $10^{-16} \mathrm{~W} / \mathrm{Hz}^{0.5}$ which can fulfill the sensitivity requirement for ground-based astronomical application. The minimum measured NEP is approximately $5.9 \times 10^{-17} \mathrm{~W} / \mathrm{Hz}^{0.5}$ at a bias voltage of $3 \mu \mathrm{V}$ and $6.4 \times 10^{-17} \mathrm{~W} / \mathrm{Hz}^{0.5}$ at a bias voltage of $12 \mu \mathrm{V}$. 
1

2

3

4

5

6

7

8

9

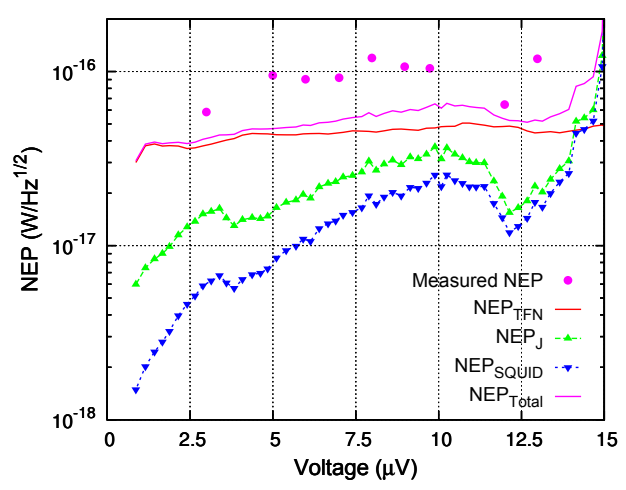

Fig. 6. Measured NEP and calculated different NEP contributions.

\section{Small heat capacity and small time constant}

The heat capacity of a thin normal metal film can be calculated according to $C_{\mathrm{n}}=\gamma T V$, where $\gamma$ is the Sommerfeld constant, $V$ the device volume, while the phonon heat capacity is negligible at temperatures below $1 \mathrm{~K}$. For superconducting film at critical temperature the heat capacity is $2.43 C_{\mathrm{n}}$. The TES is operated within the superconducting transition so that $C$ varies in between $C_{\mathrm{n}}$ and $2.43 C_{\mathrm{n}}$. In our case $C=4.5 \times 10^{-18}-10.9 \times 10^{-18} \mathrm{JK}^{-1}$ (using $\gamma=310 \mathrm{Jm}^{-3} \mathrm{~K}^{-2}$ for bulk Ti [14]). A rough estimation of energy resolution [32] using $\Delta E=\sqrt{4 k T_{0}^{2} C}$ was around $30-73 \mathrm{meV}$ (energy of around $3-7 \mathrm{THz}$ photon). This enables the applications in PNR detection of optical/IR photons.

The thermal time constant $\tau$ is $C / G$, where $C$ is the heat capacity of the detector, $G$ the thermal conductance. The extracted time constant using $G_{\mathrm{ext}}$ is thus $\tau_{\mathrm{ext}}=C / G_{\mathrm{ext}}$ $\approx 19 \mathrm{~ns}-77 \mathrm{~ns}$. For a Ti TES detector the major part of thermal conductance is the one between the electrons and phonons (electron-phonon conductance $G_{\mathrm{e}-\mathrm{ph}}$ ). The electronphonon conductance for metals within a small volume at low temperatures can be given by $G_{\mathrm{e}-\mathrm{ph}}=n \Sigma V T^{n-1}$ when the electron-phonon temperature difference $\left(\left|T_{\mathrm{e}}-T_{\mathrm{ph}}\right|\right)$ is much smaller than the phonon temperature $\left(T_{\mathrm{ph}}\right)$, where $\Sigma$ is a material-dependent constant, $V$ the volume. [32] The index $n$ can vary between 4 and 6 . Some experiments suggest that $G_{\text {e-ph }}(T)=1.56 \times 10^{9} V T^{3}$ above $300 \mathrm{mK}$ for thin Ti films on Si substrate. [14], [35] With this suggestion of approximation, the electron-phonon thermal conductance of our Ti bolometric detector is $G_{\mathrm{e}-\mathrm{ph}} \approx 3.4 \mathrm{pW} / \mathrm{K}$ and the electron-phonon relaxation time constant is therefore $\tau_{\mathrm{e}-\mathrm{ph}}=C / G_{\mathrm{e}-\mathrm{ph}} \approx 1.3 \mu \mathrm{s}$. The electron-phonon thermal conductance $G_{\mathrm{e}-\mathrm{ph}}$ is much smaller than the extracted overall thermal conductance $G_{\text {ext }}$ evaluated from measured I-V curves. This implies that there might be an extra thermal link besides electron-phonon cooling. A diffusion cooling path through electrodes might account for that.

The diffusion cooling time constant due to diffusion cooling path can be calculated according to $\tau_{\text {diff }}=L^{2} /\left(\pi^{2} D\right)$, where $L$ is the micro-bridge length and $D$ the electron diffusion constant. [21], [22] Assuming the electron diffusion constant $D=1 \mathrm{~cm}^{2} / \mathrm{s}$ [21] and a 1- $\mu \mathrm{m}$ long micro-bridge, we have an estimated diffusion time $\tau_{\text {diff }} \approx 1.0 \mathrm{~ns}$. Note that the total relaxation rate $1 / \tau=1 / \tau_{\mathrm{e} \text {-ph }}+1 / \tau_{\text {diff. }}$. If $\tau_{\mathrm{e} \text {-ph }} \gg \tau_{\text {diff }}$, we get $\tau \approx \tau_{\text {diff. }}$. However as can be seen for our detector, the $\tau_{\text {ext }}$ is in between the electron-phonon one $\tau_{\mathrm{e} \text {-ph }} \approx 1.3 \mu$ s and diffusion cooling one $\tau_{\text {diff }} \approx 1.0 \mathrm{~ns}$. It implies that the diffusion cooling is weakened to a certain degree (this leads to a greater time constant) but not well enough suppressed (if well suppressed, $\tau$ should be close to $\tau_{\mathrm{e}-\mathrm{ph}}$ ). In our detector, the area of the interface between the $\mathrm{Ti}$ micro-bridge and $\mathrm{Nb}$ electrodes is larger than the micro-bridge and the R-T curve shows that the transition temperature of contact area is not high enough to suppress the diffusion of electrons. For comparison, a sample of $25 \mu \mathrm{m} \times 25 \mu \mathrm{m} \times 51 \mathrm{~nm}$ with contact electrode area of $25 \mu \mathrm{m} \times 2 \mu \mathrm{m}$ was fabricated and tested by recording its optical response to obtain its actual relaxation time constant.

The optical response output $\delta V(t)$ of TES to input pulse can be fitted to formula as [18]

$$
\delta V(t)=A\left[\exp \left(-\frac{t-t_{0}}{\tau_{\text {decay }}}\right)-\exp \left(-\frac{t-t_{0}}{\tau_{\text {rise }}}\right)\right],
$$

where $A$ is a constant of pulse height, $t_{0}$ the incident time, $\tau_{\text {rise }}$ the rise time constant, and $\tau_{\text {decay }}$ the relaxation time constant. Averaged pulse response was recorded and fitted using the above equations and the fitting gives $\tau_{\text {rise }}=0.12 \mu \mathrm{s}$ and $\tau_{\text {decay }}=0.93 \mu$ s. The heat capacity of the large area detector is $C_{1}=3.0 \mathrm{fJ} / \mathrm{K}$ (using $\gamma T V$ ). The electron-phonon thermal conductance is $G_{\mathrm{e}-\mathrm{ph}, \mathrm{l}}=1.34 \mathrm{nW} / \mathrm{K}$ (using $1.56 \times 10^{9} V T^{3}$ ) and thus electron-phonon thermal time constant is $\tau_{\mathrm{e}-\mathrm{ph}}=$ $2.2 \mu \mathrm{s}$. Due to ETF effect the actual relaxation time constant is smaller than electron-phonon thermal time constant, [34] but still it can be seen that they are in the same order for this large area sample. This result provides a comparison to that of our small-size Ti detector. The time constant of the small-area detector was smaller than that of the larger one.

\section{CONCLusions}

In conclusion, we have fabricated a small-size Ti TES detector and characterized its electrical and thermal properties. The minimum measured electrical NEP is around $6 \times 10^{-17} \mathrm{~W} / \mathrm{Hz}^{0.5}$, which is suitable for ground-based astronomical observations. The R-T curve, the measured thermal conductance, and the thermal time constant indicate that the diffusion cooling contributes an important part in the thermal relaxation process. With high sensitivity, fast response, and low heat capacity, the small-size Ti TES detector is suitable for power detection as well as for PNR detection in astronomical applications.

\section{ACKNOWLEDGMENT}

We thank Joseph MARTINO, Damien PRELE, Fabrice VOISIN, and Michel PIAT of Laboratoire AstroParticule et Cosmologie (APC, Univ. Paris-7, France) for help with the I-V testing setup. We thank Yang LIU and Qiang ZHANG of University of Science and Technology of China (USTC, China) for help with the optical response testing setup. 


\section{REFERENCES}

[1] R. C. Jones, "The General Theory of Bolometer Performance," Journal of the Optical Society of America, vol. 43, no. 1, pp. 1-10, 1953.

[2] P. L. Richards, "Bolometers for infrared and millimeter waves," Journal of Applied Physics, vol. 76, no. 1, pp. 1-24, Jul. 1994.

[3] D. McCammon, "Thermal Equilibrium Calorimeters An Introduction," in Cryogenic Particle Detection, ser. Topics in Applied Physics, C. Enss, Ed. Berlin Heidelberg: Springer-Verlag, 2005, vol. 99, pp. 1-34.

[4] J. M. Gildemeister, A. T. Lee, and P. L. Richards, "A fully lithographed voltage-biased superconducting spiderweb bolometer," Applied Physics Letters, vol. 74, no. 6, pp. 868-870, Feb. 1999.

[5] C. L. Kuo, J. J. Bock, J. A. Bonetti, J. Brevik, G. Chattopadhyay, P. K. Day, S. Golwala, M. Kenyon, A. E. Lange, H. G. LeDuc, H. Nguyen, R. W. Ogburn, A. Orlando, A. Transgrud, A. Turner, G. Wang, and J. Zmuidzinas, "Antenna-coupled TES bolometer arrays for CMB polarimetry," Proc. SPIE, vol. 7020, p. 70201I, 2008.

[6] B. Cabrera, R. M. Clarke, P. Colling, A. J. Miller, S. Nam, and R. W. Romani, "Detection of single infrared, optical, and ultraviolet photons using superconducting transition edge sensors," Applied Physics Letters, vol. 73, no. 6, pp. 735-737, Aug. 1998.

[7] R. W. Romani, T. J. Bay, J. Burney, and B. Cabrera, "Transition Edge Cameras for Fast Optical Spectrophotometry," in High Time Resolution Astrophysics, ser. Astrophysics and Space Science Library, D. Phelan, O. Ryan, and A. Shearer, Eds. Netherlands: Springer, 2008, vol. 351, pp. 311-325.

[8] K. D. Irwin, G. C. Hilton, D. A. Wollman, and J. M. Martinis, "X-ray detection using a superconducting transitionedge sensor microcalorimeter with electrothermal feedback," Applied Physics Letters, vol. 69, no. 13, pp. 1945-1947, Sep. 1996.

[9] J. N. Ullom and D. A. Bennett, "Review of superconducting transition-edge sensors for $\mathrm{x}$-ray and gamma-ray spectroscopy," Superconductor Science and Technology, vol. 28, no. 8, p. 084003, 2015.

[10] J. E. Austermann, K. A. Aird, and et al., "SPTpol: an instrument for CMB polarization measurements with the South Pole Telescope," Proc. SPIE, vol. 8452, p. 84521E, 2012.

[11] W. S. Holland, D. Bintley, E. L. Chapin, A. Chrysostomou, G. R. Davis, J. T. Dempsey, W. D. Duncan, M. Fich, P. Friberg, M. Halpern, K. D. Irwin, T. Jenness, B. D. Kelly, M. J. MacIntosh, E. I. Robson, D. Scott, P. a. R. Ade, E. Atad-Ettedgui, D. S. Berry, S. C. Craig, X. Gao, A. G. Gibb, G. C. Hilton, M. I. Hollister, J. B. Kycia, D. W. Lunney, H. McGregor, D. Montgomery, W. Parkes, R. P. J. Tilanus, J. N. Ullom, C. A. Walther, A. J. Walton, A. L. Woodcraft, M. Amiri, D. Atkinson, B. Burger, T. Chuter, I. M. Coulson, W. B. Doriese, C. Dunare, F. Economou, M. D. Niemack, H. a. L. Parsons, C. D. Reintsema, B. Sibthorpe, I. Smail, R. Sudiwala, and H. S. Thomas, "SCUBA-2: the 10000 pixel bolometer camera on the James Clerk Maxwell Telescope," Monthly Notices of the Royal Astronomical Society, vol. 430, no. 4, pp. 2513-2533, Apr. 2013.

[12] B. S. Karasik, B. Delaet, W. R. McGrath, J. Wei, M. E. Gershenson, and A. V. Sergeev, "Experimental study of superconducting hotelectron sensors for submm astronomy," IEEE Transactions on Applied Superconductivity, vol. 13, no. 2, pp. 188-191, 2003.

[13] B. S. Karasik, D. Olaya, J. Wei, S. Pereverzev, M. E. Gershenson, J. H. Kawamura, W. R. McGrath, and A. V. Sergeev, "Record-Low NEP in Hot-Electron Titanium Nanobolometers," IEEE Transactions on Applied Superconductivity, vol. 17, no. 2, pp. 293-297, Jun. 2007.

[14] B. S. Karasik, S. V. Pereverzev, D. Olaya, J. Wei, M. E. Gershenson, and A. V. Sergeev, "Noise Measurements in Hot-Electron Titanium Nanobolometers," IEEE Transactions on Applied Superconductivity, vol. 19, no. 3, pp. 532-535, Jun. 2009.

[15] B. S. Karasik, A. V. Sergeev, and D. E. Prober, "Nanobolometers for THz Photon Detection," IEEE Transactions on Terahertz Science and Technology, vol. 1, no. 1, pp. $97-111$, Sep. 2011.

[16] B. S. Karasik and A. V. Sergeev, "THz hot-electron photon counter," IEEE Transactions on Applied Superconductivity, vol. 15, no. 2, pp. 618-621, Jun. 2005

[17] D. F. Santavicca, B. Reulet, B. S. Karasik, S. V. Pereverzev, D. Olaya, M. E. Gershenson, L. Frunzio, and D. E. Prober, "Energy resolution of terahertz single-photon-sensitive bolometric detectors," Applied Physics Letters, vol. 96, no. 8, pp. 083 505-083 505-3, Feb. 2010.

[18] D. Fukuda, G. Fujii, A. Yoshizawa, H. Tsuchida, R. Damayanthi, H. Takahashi, S. Inoue, and M. Ohkubo, "High Speed Photon Number Resolving Detector with Titanium Transition Edge Sensor," Journal of Low Temperature Physics, vol. 151, no. 1, pp. 100-105, 2008.
[19] C. Portesi, E. Taralli, L. Lolli, M. Rajteri, and E. Monticone, "Fabrication and characterization of fast TESs with small area for single photon counting," IEEE Transactions on Applied Superconductivity, vol. 25, no. 3, pp. 1-4, Jun. 2015 .

[20] W. Zhang, J. Q. Zhong, W. Miao, Z. Wang, D. Liu, Q. J. Yao, S. C. Shi, T. J. Chen, and M. J. Wang, "Electrical Characteristics of Superconducting Ti Transition Edge Sensors," Journal of Low Temperature Physics, vol. 184, pp. 11-16, Oct. 2016.

[21] B. S. Karasik, K. S. Il'in, E. V. Pechen, and S. I. Krasnosvobodtsev, "Diffusion cooling mechanism in a hotelectron $\mathrm{NbC}$ microbolometer mixer," Applied Physics Letters, vol. 68, no. 16, pp. 2285-2287, Apr. 1996.

[22] A. Skalare, W. R. McGrath, B. Bumble, H. G. LeDuc, P. J. Burke, A. A. Verheijen, R. J. Schoelkopf, and D. E. Prober, "Large bandwidth and low noise in a diffusioncooled hotelectron bolometer mixer," Applied Physics Letters, vol. 68, no. 11, pp. 1558-1560, Mar. 1996.

[23] L. Lolli, E. Taralli, M. Rajteri, T. Numata, and D. Fukuda, "Characterization of Optical Fast Transition-Edge Sensors With Optimized Fiber Coupling," IEEE Transactions on Applied Superconductivity, vol. 23, no. 3, pp. $2100904-2100904$, Jun. 2013.

[24] K. Irwin, S. Nam, B. Cabrera, B. Chugg, G. Park, R. P. Welty, and J. Martinis, "A self-biasing cryogenic particle detector utilizing electrothermal feedback and a SQUID readout," IEEE Transactions on Applied Superconductivity, vol. 5, no. 2, pp. 2690-2693, 1995.

[25] R. Cantor, L. P. Lee, A. Matlashov, and V. Vinetskiy, "A low-noise, twostage DC SQUID amplifier with high bandwidth and dynamic range," IEEE Transactions on Applied Superconductivity, vol. 7, no. 2, pp. 3033-3036, Jun. 1997.

[26] "pcSQUID" TM advanced PC-based SQUID control electronics of STAR Cryoelectronics," http://www.starcryo.com/pcsquid/.

[27] F. N. Hooge, "1/f noise sources," IEEE Transactions on Electron Devices, vol. 41, no. 11, pp. 1926-1935, Nov 1994

[28] G. P. Zhigal'skii, "1/ f noise and nonlinear effects in thin metal films," Physics-Uspekhi, vol. 40, no. 6, p. 599, 1997.

[29] D. Drung, C. Hinnrichs, and H.-J. Barthelmess, "Lownoise ultra-high-speed dc SQUID readout electronics," Superconductor Science and Technology, vol. 19, no. 5, p. S235, May 2006.

[30] "SQUID systems of Magnicon GmbH," http://www.magnicon.com/squid-systems/.

[31] H. Ekström, B. S. Karasik, E. L. Kollberg, and K. S. Yngvesson, "Conversion gain and noise of niobium superconducting hot-electronmixers," IEEE Transactions on Microwave Theory and Techniques, vol. 43, no. 4, pp. $938-947,1995$.

[32] K. D. Irwin and G. C. Hilton, "Transition-Edge Sensors," in Cryogenic Particle Detection, ser. Topics in Applied Physics, C. Enss, Ed. Berlin Heidelberg: Springer-Verlag, Jan. 2005, vol. 99, pp. 63-150.

[33] J. C. Mather, "Bolometer noise: nonequilibrium theory," Applied Optics, vol. 21, no. 6, pp. 1125-1129, Mar. 1982.

[34] K. D. Irwin, "An application of electrothermal feedback for high resolution cryogenic particle detection," Applied Physics Letters, vol. 66, pp. 1998-2000, Apr. 1995

[35] J. Wei, D. Olaya, B. S. Karasik, S. V. Pereverzev, A. V. Sergeev, and M. E. Gershenson, "Ultrasensitive hot-electron nanobolometers for terahertz astrophysics," Nature Nanotechnology, vol. 3, no. 8, pp. 496500,2008 